\title{
BMES 2009 Distinguished Achievement Lecture Award
}

\author{
BMES Annual Meeting October 9, 2009
}

(Received 23 December 2009; accepted 25 December 2009; published online 3 February 2010)

Bill Hawkins assumed the role of Chief Executive Officer of Medtronic, Inc. in August 2007 and became Chairman of the Board in August 2008. Hawkins was named President and Chief Operating Officer in May 2004 after joining Medtronic as Senior Vice President and President of Medtronic's Vascular business in January 2002. Hawkins joined Medtronic from Novoste Corp., where he had been President and Chief Executive Officer since 1998. Earlier positions included Corporate Vice President and President of the Sherwood Davis and Geck organization of American Home Products; President of the Ethicon EndoSurgery organization of Johnson \& Johnson; President, Devices for Vascular Intervention and President U.S. Operations, for Guidant Corp.; and several increasingly responsible executive positions culminating in the presidency of the IVAC organization for Eli Lilly \& Co. He began his medical technology career with Carolina Medical Electronics in 1977. Hawkins received his Bachelor of Science degree in electrical and biomedical engineering from Duke University in 1976 where he also conducted medical research in pathology. He received a Master of Business Administration Degree from the Darden School of Business, University of Virginia, in 1982. Hawkins is a member of the Board of Visitors of the Engineering School of Duke University and the Guthrie Theatre Board. His Distinguished Achievement Lecture Award is provided verbatim and as delivered on October 9, 2009.

Good morning. It is great to be here with you today. I am truly honored and grateful for this recognition. This award holds special meaning for me since I graduated from Duke 33 years ago with a dual degree in Biomedical and Electrical Engineering. At that time few people knew what a Biomedical Engineer was. Companies recruiting on campus did not know how to specify Biomedical, and hence, opportunities were limited. Well, today things are different, as this has become one of the most popular and sought-after fields, attracting a diverse mix of genders and nationalities to its ranks. I want to take this opportunity first to thank the members of the Biomedical Engineering
Society, and particularly its leadership, past and present, for their many efforts in elevating this still young discipline and making it one of the most important sectors of engineering. Biomedical engineering serves people where it is most personal - their health and well being. It has become a field that is changing lives for the better - every day, all around the world.

Looking back over the last 33 years, I am amazed by what has been achieved in advancing a discipline that has prepared so many to do so much. I remember my first professional experience with Carolina Medical, a small medical technology company located near Winston Salem, North Carolina. I was hired a year after graduating from Duke to work in sales. There were only four of us covering the entire United States. Our product was an electromagnetic blood flow meter used during open heart surgery. Working in the OR, I watched as surgeons hooked up early versions of heart lung bypass systems and then shocked the patient's heart, sending them into asystole so they could perform an anastomosis on an arrested heart. Afterwards, they used my flow meter to measure blood flow in the new grafts. A year later, we pioneered the first-ever use of ultrasound to map flow in the carotid arteries.

Today, we navigate the vasculature with tiny catheters deploying stents, eliminating the need to open a patient's chest, and ultrasound is now the standard of care and used to noninvasively diagnose a wide range of conditions. How far we have come in such a brief period!

Today I am proud to be the Chairman and CEO of Medtronic, a company that is leading the development of many of today's and tomorrow's most innovative biomedical technologies.

Medtronic was founded in 1949 in Minneapolis as a medical equipment repair company, by Earl Bakken, an electrical engineer, and his brother-in-law, Palmer Hermundslie.

Today, we're the world's largest independent medical technology company. We employ about 38,000 talented people around the world; our 9,000 engineers and thousands of highly skilled clinical and sales 
professionals serve physicians and patients in more than 120 countries.

Our unflagging commitment to advancing state-ofthe-art technology is evidenced by the $\$ 1.4$ billion we invested last year in research and development.

Many of you may know that Medtronic's roots are firmly entrenched in treatments for cardiac and vascular disease, but today we are a leader in chronic disease management, focusing also on the treatment of diabetes, neurological disorders and pain; spinal and musculoskeletal disorders; urologic and digestive disorders; and, ear, nose, and throat conditions.

Every 4.8 seconds someone, somewhere has their life improved as a direct result of a Medtronic product, which itself is the direct result of an engineering or scientific idea that we have translated into a medical technology. That's our business - creating innovations that restore, enhance, and save lives.

I cannot imagine a more exciting field to be a part of; one that rewards our natural curiosity and creativity with the satisfaction that what we do can have a major and lasting impact on the health of the world's nations, communities, families, and individuals.

What's more exciting is to imagine what the future might hold for the amazing technologies being developed today; technologies that tomorrow might restore people to fuller lives and even provide cures for some of the world's most difficult-to-treat chronic diseases.

Imagine for a moment, a world...

Where tomorrow... a man with epilepsy has a dimesized implanted device that senses the onset of a seizure and then delivers a precise stimulus to a pre-specified target in the brain, terminating what otherwise may have been a debilitating, convulsive seizure.

This tomorrow is already being realized today, through our development of closed-loop auto-feedback systems, and builds on our ground-breaking work using deep brain stimulation for Parkinson's disease, dystonia, Tourette's Syndrome, essential tremor, and obsessive compulsive disorder.

Imagine a world where tomorrow... with the prick of a finger, a few drops of blood will identify and diagnose a broad array of potential diseases and conditions based on the patient's genetic profile; conditions that typically would only manifest themselves in their later stages, in many cases after it was too late to receive effective treatment.

This tomorrow is being realized today as the promise held by the mapping of the human genome is being translated into sophisticated diagnostics. Now, physicians can identify both the diseases and the treatment an individual will best respond to based on their unique genetic profile - making personalized medicine, truly personalized.
Imagine a world where tomorrow...many of the world's most debilitating chronic diseases are managed systematically, comprehensively, and even remotely, as patients, physicians, and hospitals are connected in a seamless web of wireless technology. This will enable real time disease monitoring, diagnosis, and intervention, empowering patients to manage their own disease and physicians to make diagnosis and treatment decisions more quickly and more effectively, unburdening an increasingly financially strapped and inefficient healthcare system.

This tomorrow is being realized today through remote monitoring technologies that are being integrated into our therapies for diabetes, heart disease, and neurological disorders.

And imagine a world where tomorrow...many of today's invasive surgical procedures will be routinely performed on an outpatient basis, made possible by advances in minimally-invasive technologies that allow physicians to leverage the body's vascular highway or alimentary tract to perform complicated procedures on vital organs and systems, reducing the risks and complications of surgery and eliminating the costs associated with in-patient hospitalizations.

This tomorrow is being realized today through the percutaneous transcatheter technologies that are poised to revolutionize the way we approach surgery, shifting the paradigm from invasive to minimallyinvasive to non-invasive.

Recently, Medtronic acquired a ground-breaking technology called CoreValve, which is making this tomorrow a reality today, right now.

The accomplishment of bringing to patients useful technologies that once seemed possible only in theory is one of the greatest rewards of my job.

Since 1949-60 years ago - our industry has made remarkable contributions to improving human health. But if we look further into the future, what might the next wave of revolutionary advances in disease management look like? Will they require any interaction on the part of the patient? Will they even be visible? Or might they operate invisibly, integrating into the body to become a seamless, organic extension of it?

Tissue engineering, as an example, has produced some dramatic and tantalizing early results. Researchers in Boston have repaired portions of defective urinary bladders in children using harvested autologous cells to replace damaged sections. Others have found a way to use cultured bladder cells to reconstruct larger, damaged portions of a whole bladder. And researchers at the University of Minnesota have demonstrated the ability to grow a beating heart in the laboratory using stem cells and tissue engineering. Breakthroughs like this may one day even eliminate today's lengthy transplant waiting 
lists, where many patients in desperate need die waiting in line.

These are just a sampling of what science and technology are making possible today. It's these, and a myriad of other similar advancements, that lead me to believe we're on the cusp of major transformation and a great leap forward, made possible by my colleagues in the medical technology industry, and those of you here in this room.

We are evolving from palliative therapies that primarily alleviate the symptoms of disease, to restorative therapies in which replication of the natural system will unquestionably yield better results and perhaps even cures.

If our history and experience are any indication, the progress we're making today toward these amazing tomorrows will not just be fully realized, but pushed far beyond, by the biomedical engineering community.

And although biomedical engineering is by definition an interdisciplinary field, realizing the promise of tomorrow will require that we expand the interaction and interdependencies both within and between other academic disciplines and industries.

As I will discuss later, it will also mean that we will have to develop stronger linkages to other sectors that profoundly influence our work and our ability to treat patients.

Put differently, we need to create better ways to share and collaborate; ways that will ultimately unleash the great potential enabled by the advancement and convergence of the engineering, life, and information sciences.

The theme of this year's Biomedical Engineering Society meeting, "Bridging the three rivers of biology, engineering, and medicine," is a very apt description of how our field and our industry is evolving.

As a case in point: yesterday's Pritzker Distinguished Lecturer, Donald Ingebar, comes from the field of "biologically inspired engineering," a new field that is giving us important insights into how nature has designed living systems; insights that will provide us with the practical tools that will help innovate the next wave of great biomedical advancements. It is this discipline, and others, that were unimagined when I was a student at Duke 33 years ago.

Yet, this kind of academic innovation does not surprise me. Biomedical engineering by its very nature is inclusive, integrative, and collaborative. It has always thrived on the cross-pollination of multiple disciplines and fields.

It could even be argued that the convergence of the engineering, life, and information sciences was in many ways fueled by biomedical engineering, making us both the progenitors and the benefactors of its fruits.
That said, if we are going to truly realize the full potential of what we are doing today we must broaden our focus to insure that these new technologies are not only commercialized, but accepted by mainstream medicine as the standard of care.

To accomplish this, we must now consider additional factors that influence our ability to translate concepts into solutions that become the standard of care in our field. To this end, I want to discuss what I refer to as Translational engineering or Translation.

I know this term has gained traction in academia, in large measure due to the emphasis placed on translational research by the NIH and NSF. However, a more expansive view of translation has taken on a new imperative in industry as well.

This broader view of translation calls upon us to re-conceptualize the very essence of how we think, teach, train, and work.

It requires that we commit ourselves to a deliberate and thoughtful effort to transform our field and expand our collaboration beyond our traditional partners.

Our challenge is this: How do we translate more than 60 years of rapid technology advancement and interdisciplinary convergence into the powerful future we imagined earlier?

A good place to start is by examining past successes for the technologies and environmental conditions that made them possible. Similarly, we should also examine those that might serve as potential barriers to progress and innovation.

By doing this, we will better understand the gaps that we need to fill - whether they be technical, scientific, organizational, political, or cultural. We can then develop a pathway forward to translate concepts more rapidly into practical, life-enhancing, and life-saving therapies.

In order for us to best understand what we need to do today to realize the possibilities of the future, let's imagine, if you will, reverse engineering the future of medical technology. Working backwards we can then determine what we need to do to address the barriers going forward that are likely to constrain our capabilities.

The early years of our industry were characterized by rapid, albeit somewhat uncoordinated, technology development across multiple discrete disciplines. These technologies would ultimately become the building blocks that form the foundation for many of today's most advanced medical devices.

The 1940s and 1950s saw the development of intraocular lenses, the synthetic aortic graft and the cardiac pacemaker. In the 1960s followed the cageand-ball heart valve.

By the 1970s and into the 1980s, these advancements progressed to a much broader range of therapies to 
treat chronic conditions of the spine, nervous, and endocrine systems and pain.

The 1980s saw electrical stimulation applied to essential tremor and other chronic conditions of the brain and nervous system, the emergence and expansion of diagnostic and remote monitoring technology, and the first drug delivery devices used for diabetes, pain management, and disorders like spasticity.

It was the convergence of advancements in engineering, life, and information sciences that made these achievements possible.

In the engineering sciences, there were major advancements in several areas including:

- Ultra low-power microprocessing technologywhich provided the advanced data processing, communications, and telemetric capabilities that made devices smarter, easier to use, and of better quality

- An array of novel biomaterials - which provided materials capable of withstanding the incredibly harsh environment found within the body and its systems, giving us improved biocompatibility, greater biointeractivity, and better reliability

- Miniaturization - which enabled the development of smaller componentry that enabled us to reduce devices from the size of a hockey puck to the size of pocket change

- New power sources - the transition from mercury chemistry to lithium provided improved energy and power density and also increased device reliability and longevity

- Imaging and optical technologies - which have provided the tools and capabilities to see the human anatomy and its ailments-handily and in robust detail. Technologies like ultrasound, CAT, and MRI scans have been critical to allowing us to visualize and navigate the body without opening it

The life sciences have given us:

- Biotechnology - which has enabled us to leverage advancements in cell and molecular biology to derive treatments for some of the world's most acute and deadly diseases, including cancer, rheumatoid arthritis, and autoimmune disorders

- Molecular diagnostics - which is leveraging the vast warehouse of information contained in the human genome to revolutionize the way we diagnose and treat disease

- Biologically inspired engineering - a field I mentioned before, which applies our understanding of biology to create novel, human-engineered systems
And finally, the information sciences have given us:

- Communications and connectivity_providing better, faster, and more reliable information transferred both from, and between, devices

- Connected care and eHealth - a technology enabled model that seamlessly connects patients, providers, payers and others

In the 1990s, our industry drove an exponential expansion of the indications for our therapies, and correspondingly, the number of people who benefitted from them.

Electrical stimulation was being used to treat Parkinson's disease, heart failure and overactive bladder.

Innovative mechanical devices were developed for otherwise difficult-to-treat conditions, such as blocked coronary arteries, abdominal aortic aneurysms, and lumbar disk herniations.

Drug delivery expanded into the treatment of chronic and non-malignant pain, and the diagnostic and remote-monitoring technologies used in heart disease were now being developed for use in diabetes.

The rapid progress of the 1990s was driven by the convergence of the many branches of science, medicine, and engineering. This convergence created much closer, cross-disciplinary collaboration among the fields and disciplines that once worked more or less independently.

Convergence drove a number of critical developments, both technical and scientific in nature, including:

- Regenerative medicine-which gave us the amazing ability to replace damaged tissue with biological equivalents that can encourage the body to maintain, repair or enhance itself

- Molecular diagnostics - which unlocked the power of genomics, proteomics, and other biologicallybased principles that help us better diagnose disease and personalize patient therapies

- Combination devices that merge device technologies with those of pharmaceuticals or biologics, offering site-specific delivery of a drug or biotherapeutic to optimize therapy, reduce side effects, and achieve clinical results that may not be possible by traditional delivery mechanisms

- Combination devices that merge information technologies and medical technologies, enabling us to remotely monitor patients at risk of SCD or patients suffering from heart failure

Clearly, a powerful industry had grown in an amazingly short period of time. Today the medical device industry is a unique American success story. We generate revenues of almost 200 billion dollars 
globally, comprise more than 15,000 companies in the U.S. alone, and employ more than 400,000 people around the world. By almost any measure we are an industry that we need to nurture and cultivate.

As we look forward, our challenge is how to translate the new technologies created through convergence into the social benefits we know are possible.

People often ask me the question that I imagine many CEOs get these days, "what keeps you up at night?" Well, I'll tell you. When I think about the future, and the vast possibilities enabled by our technologies, I worry less about the technological or scientific challenges that must be overcome, and more about the external environmental challenges that must also be overcome for us to realize this tremendous societal benefit.

Our broader view of translation is about harnessing the forces of technology and convergence, put in motion some 60 years ago, to transform the practice of medicine and move from the mere palliative to the restorative by enabling customization of therapies tailored to specific individuals, by empowering patients to take an active role in managing their own care, and by lowering the complexity of therapy while maximizing efficacy. This will create long-term solutions that will dramatically improve human health and drive healthcare costs down.

In many ways, translation is characterized more by human endeavors than by technological or scientific endeavors. Translation requires that we move beyond the traditional application of biology, engineering, and medicine, and to understand and actively shape the environmental factors that will largely determine when, if and how technologies reach patients. These challenges have much more to do with approval, delivery, access, and affordability than with technology itself, and in many ways are much more vexing and unfamiliar. These are problems that we as engineers are not traditionally trained to navigate.

There are four areas of our external environment that each pose challenges to the translation of technology: The cultural environment, the legal, the regulatory, and the public policy environments.

A good example of the challenges we face in our current cultural environment is patient and public expectations regarding the quality, reliability, and effectiveness of our technologies. These expectations are really indicative of a much broader cultural bias toward nearly total risk aversion. Ironically, in this regard we may be the victims of our own success.

In the 1970s and 1980s, there was great public and physician enthusiasm for the device technologies that were now being used with increasing frequency to restore patients to fuller, more active lives. Technologies that for many were the only solution for their disease or condition.
As an industry committed to human health and well-being, the safety of our therapies was of paramount concern - and we took very seriously the efforts and investments we made with each new device iteration to produce safety and quality advancements. Taken collectively, these efforts represent one of the most sustained and successful quality commitments undertaken by any industry. The end result was a substantial increase in the level of overall product quality to impressive, albeit still imperfect, levels.

Let me be clear: Nothing is more important to us than quality. Our challenge is how do we continue to deliver innovative, high-quality products to an achievable standard that appropriately balances benefits against possible risks? The challenge here is one around risk management.

This is not a challenge that can be "engineered" away. This is a challenge that demands of us a much broader range of thinking and engagement in disciplines and areas that might seem, at first, entirely unrelated to engineering and science.

Fortunately, the critical and analytical thinking skills instilled by our traditional training equip us well to innovate around the notion of risk management, much as we do in the laboratory. But make no mistake - action will be required to increase the public's understanding of both the promise of technology, as well as its limitations.

The next area has to do with our legal environment.

The tort system we have in the United States is not always aligned with the best interests of patients and society as a whole, and can distort clinical decisionmaking, requiring care givers to do things for the wrong reasons.

The current healthcare debate has highlighted the potential our legal system has to stifle innovation and increase costs from litigation and overutilization driven by "defensive medicine."

The threat of excessive litigation has diverted resources away from innovation and sometimes discouraged companies from introducing highly sophisticated and beneficial technologies to the clinical community. This must be changed for us to realize the full societal benefit and impact of our innovation.

We are also facing an increasingly complex and costly regulatory environment.

In many ways, this is a direct result of the other environmental factors I just mentioned. However, it is also an area that, perhaps even more so than others, will require strong leadership, increased collaboration and sustained confidence-building efforts between industry, academia, the public, and the agencies that oversee the regulatory process.

We all share the common goal of improving human health and welfare. And by virtue of this commonality, 
there exists great potential and opportunity for us to create a regulatory environment that serves the best interests of patients by both protecting their safety and supporting the innovation that so many will benefit from.

We need to work together with the clinical and regulatory community to develop protocols that more effectively and efficiently accomplish the legitimate and beneficial goals of regulation. We must insure that our regulatory systems bring valuable, safe, and high-quality new technologies to patients in a timely and costeffective manner. We must work together in innovating new models for evaluating post market performance.

Without collaboration and consensus around common goals, product cycle times will continue to increase and the introduction of newer, improved and life-saving technologies will be delayed.

From an academic perspective, the field of regulatory "science" has been outpaced by advancements in technology science; a situation that I believe can be vastly improved by providing formal education and training to current and future generations of biomedical engineers. For instance, what lessons can be learned from comparative studies of regulatory processes outside of the United States? Is it coincidental that most medical innovation is first introduced to the marketplace outside of the United States? These are questions that are worth exploring.

As you can imagine, these challenges have had a ripple effect on the public policy environment, posing potentially serious impediments to innovation.

Current efforts to reform our healthcare system have taken center stage in the political arena. Throughout the discussions and the debate, Medtronic and many of our industry counterparts have been actively engaged with policymakers and the White House regarding elements of healthcare reform that we believe are critical for the long term success of our healthcare system.

We support the idea that all Americans should have access to affordable health coverage. We also support the concept of comparative effectiveness studies that require products to demonstrate a proven benefit for their intended patient populations.

However, meaningful healthcare reform will not be achieved if a primary focus is placed on cost alone. It is critical that any proposal consider the quality of the treatment being provided and the need to provide increased access to the best treatment options available.

By reducing hospitalizations, decreasing procedure times, empowering patients to take control of their disease management and most importantly-improving long term outcomes-we believe that medical technology can be a significant part of the solution for healthcare reform.
Furthermore, we have strong concerns about the level of the excise tax on the medical device industry that is currently being proposed, as it will divert resources away from where they are needed most-valuable investment in innovation. This could also impact jobs in what is one of the most vibrant sectors of the economy.

And finally, a few words on an issue that has gained quite a bit of media attention in the past several months: Physician collaboration and conflict of interest disclosure.

There is, today, an active Congressional and media debate about how healthcare companies work with the clinical community to innovate, to teach and to train.

I cannot emphasize enough how vital collaboration with physicians is for innovation and the health of the medical technology industry. Unlike pharmaceutical companies, who typically innovate from "bench to bedside" we use a different model - one that I call from "bedside to bench to bedside" - that both starts and ends with the physician, in an ongoing and iterative cycle that fuels rapid and essential product and procedure innovation.

Innovation in medical technology requires a close collaboration between physicians and industry. Physicians play a critical, multi-faceted role in our ability to translate innovative ideas into medical products. They are inventors who see the "flash of light" of a new idea. They provide vital clinical insight regarding the suitability of a new technology to actual patients. They suggest improvements to ideas in development and to existing products. They delineate patient selection criteria and indications for use. They devise surgical techniques necessary for safe device implantation. They conduct safety and effectiveness clinical trials to support regulatory submissions. They assess long-term performance. And, often greatly under-appreciated, they teach their colleagues how to correctly harness technological innovations for the benefit of indicated patients. While we would like to think application of medical devices is simply plug-and-play, it isn't. In short, expert physicians are an integral part of our innovation team, not just customers for our products.

However, we also understand the potential for conflicts of interest to occur and recognize our responsibility to insure that our relationships minimize potential conflicts.

Medtronic, as the industry leader, has played a strong, ongoing leadership role in working to create new standards for financial transparency and conflict of interest disclosure - and we will continue to do so.

But, it is important to remember that no matter what new changes and standards are put in place, we must be steadfast in our unyielding commitment to do what is right for the patient, which will ALWAYS be our top priority. 
So as you can see, these challenges in our external environment are becoming more complex and ever more important to our success as biomedical engineers. It will be our ability to drive change within these environments that will determine whether we unleash the potential of our innovative power, or stifle and ultimately cripple it.

You may be asking yourselves, "What can I do as an academic, an educator, an engineer - an innovator-to support and influence efforts in these critical areas?"

Research that advances both our theoretical knowledge and our practical application of science, engineering, and medicine, including novel ways in which to combine the advances from each, remains critical and central to our collective mission.

But as I have said, in today's world - the world which has become our laboratory-scientific and technological advancement is only one part of the equation.

There is no more natural a place for the "practice" of translation to take hold and become institutionalized than in the world of academia. I firmly believe our academic institutions must play a leading role in this effort.

How, for instance, will we prepare the innovators of tomorrow for the challenges of navigating today's complex regulatory environment? Today's Medicare and reimbursement environment? Today's healthcare reform environment? What kind of curriculum might be required to accomplish this? How can we augment the current curriculum to bridge the knowledge gap between technology development and the external environments that must be understood and shaped for translation to occur?

Put more broadly, how will we train tomorrow's innovators to view technology development as but a single piece of a much broader continuum, one that begins with technology, but also includes the broader external environment?

It is in this area where our academic institutions need to play an increasingly vital role in training future generations how to successfully innovate within complex and global healthcare systems, often in collaboration with the companies and physicians on the "front lines" of medical innovation.

Lawton Burns, Professor of Healthcare Systems and Director of the Center for Health Management and Economics at The Wharton School said it well in the preface of his book The Business of Healthcare Innovation.

He suggests that the purpose of this kind of knowledge is "not so much to educate students about their own sphere of activity, but rather to educate them about other sectors that are increasingly interdependent with their own."

And to this we should add the institutional web that forms the connective tissue between these sectors and our patients.

Finally, let me end on a personal note - a personal call to action and invitation for each of you here in this room today.

I believe that our industry sits upon an inflection point that requires us to examine and expand the roles we play within the broad range of environments that comprise the world that is our lab. In doing so, we must hold ourselves accountable for taking action to influence the outcomes of the grand debates upon which the great promise of our future rests.

In closing, I ask you to consider how your contribution to biomedical engineering can extend beyond the traditional aspects of engineering and science. You have a unique opportunity to inform others regarding the benefits and risks of biomedical technology - its enormous promise as well as its limitations. Effective translation of this technology into societal benefit depends on your thoughtful participation in the debates taking place today in our external environment. For many of you, this is a new role, but an increasingly critical component of what it will mean to be a biomedical engineer of the future. Your engagement in this broader arena will enable us to achieve the tomorrows that our great technological advancements make possible.

Thank you.

William A. Hawkins

Medtronic, Inc. 